\title{
Improved Energy Detector for Spectrum Sensing Using Neuro-Fuzzy Double Threshold Technique
}

\author{
Barnali DEY ${ }^{1}$, Ashraf HOSSAIN ${ }^{1}$, Valentina. E. BALAS ${ }^{2 *}$, R.N. BERA ${ }^{3}$ \\ ${ }^{1}$ Department of ECE, National Institute of Technology Silchar, Assam, India, e-mail: ashraf@ece.nits.ac.in, \\ barnali.mou.dey@gmail.com. \\ ${ }^{2}$ Department of Automation and Applied informatics, Aurel Vlaicu University of Arad, 310130, Romania, \\ valentina.balas@uav.ro (*Correspondingauthor) \\ ${ }^{3}$ Department of ECE, Sikkim Manipal Institute of Technology, Sikkim, India, e-mail: rbera@smit.smu.edu.in
}

\begin{abstract}
This paper reports a modified and computationally efficient energy detection method for spectrum sensing in cognitive radio (CR) system utilising hybrid soft computing paradigms. The proposed soft computing method uses artificial neural network and fuzzy logic for learning and decision making as a solution to the problems when CR system is subjected to threshold uncertainty and uncertainties in noise variance. The proposed soft computing method should be referred thereafter as Neuro-Fuzzy double threshold (NFDT) technique. The developed technique utilizes the concept of double sensing credibility while formulating the fuzzy decision rule and artificial neural network to learn the threshold function. Through simulation results it is validated that proposed method leads to overall improved probability of detection compared to conventional and existing methods.
\end{abstract}

Keywords: Spectrum Sensing, Double threshold, Energy detector, Cognitive Radio (CR), Neuro-Fuzzy technique.

\section{Introduction}

The unprecedented growth in the demand for the usage of radio frequency spectrum by the wireless communication users has given rise to the concept of Cognitive Radio (CR) [13]. In a cognitive radio system (or network), a cognitive user (otherwise called secondary user (SU)), opportunistically access the allocated spectrum band of the licensed user also known as the primary user (PU) without disturbing them. The whole process of how a cognitive radio functions is explained by the $\mathrm{CR}$ cycle in [16].

The implementation of various features of cognitive radio as outlined in [16], spectrum sensing is one of the most important functionality [31]. It involves detection of PU signal by the CR device which consequently leads to utilize the vacant radio spectrum band effectively.There are various methods of spectrum sensing for cognitive radio system (or network) like, Energy Detection [17], Matched filter detection [6] and cyclostationary feature detection [8]. Energy Detection is the most popular technique owing to its low computational complexity and simple structure for implementation. The decision making principle for energy detection method is based on binary hypothesis technique [14]. The main disadvantage of adoption of binary hypothesis in energy detection method is the use of single threshold value that gives rises to several other drawbacks, (i) poor sensing of PU signal at low SNR (Signal-to-Noise-Ratio) (ii) inaccurate sensing result for non-Gaussian noise samples (iii) combined effect of (i) and (ii) which leads to imprecise computation of the threshold value, thereby leading to wrong measurement of $\mathrm{P}_{\mathrm{D}}$ and $\mathrm{P}_{\mathrm{FA}}[1]$, (iv) high computational complexity at low SNR values.

To circumvent the disadvantages highlighted above researchers have attempted to improve the sensing performance of conventional energy detection method under low SNR condition by adopting various modifications owing to its computational advantage. One such modification put forward in [24] is to divide the threshold value into two parts as shown in Fig. 1. The idea behind using two threshold values instead of one is to accurately decide the presence or absence of PUs by SUs-referred as double thresholding technique. In the present work, the idea of [24] is extended to a CR system with one SU and one PU with no provision of fusion centre and modifications in dividing the thresholds. The disadvantage of the method proposed in [24] for spectrum sensing is that, when energy values fall in a region between two threshold values a confusion arises in taking decision in this region - known as "confused region" or "no decision" region. Usually a no decision is taken by $\mathrm{CR}$ in this region thus leading to 'sensing failure'. This problem has been tackled by various soft and hard decision schemes using a Fusion Centre for CR network.

To the best of the author's knowledge the problem has not been tackled effectively at the local CR level (i.e., at the individual CR). 


\section{Related Work}

The inherent limitation of conventional energy detector is unreliability and inaccuracy in sensing due to the presence of uncertainty in noise distribution under practical scenario [12] and thereby using single threshold in the hypothesis testing. To mitigate this problem of noise uncertainty, authors in $[25,15,5]$ used the concept of double threshold (introduced above) in a cooperative sensing environment for CR network. The idea of hierarchical quantization has been exploited in [6] at the local CR level. In [26] improvement has been attempted using single threshold but in a cooperative sensing environment. Alternatively different from the above approaches researchers have tried to solve this limitation of energy detector by adopting soft decision method using Fuzzy logic [18, 27, $19,20,9]$ as there is great deal of ambiguity, uncertainty or fuzziness in the 'confused region' as shown in Figure 1 for a cooperative sensing scenario. The disadvantages cooperative based sensing are the increased hardware overhead as well as sensing latency.

The contribution of the present method is improved sensing performance at the local CR level instead of adopting cooperative sensing technique under two distinct uncertain scenarios, (a) uncertainty in threshold computation and (b) uncertainty in noise variance estimation. To accomplish these objectives, following modifications are adopted, (i) use function approximation capability of neural network to learn the threshold function as given in (7) (ii) divide the threshold in (7) into two parts to handle the uncertainties described above in (a) and (b) and (iii) to handle the ambiguity in the "no decision" region a new fuzzy decision rule base along with a fuzzy threshold criterion has been proposed. To the best of the author's knowledge such hybrid Neuro-Fuzzy based scheme has not been proposed so far to improve sensing performance at the local CR level for two different uncertain situations.

\section{System Description}

In this section necessary background of the conventional energy detector with single threshold and subsequently the motivation to evolve double threshold energy detector are presented.

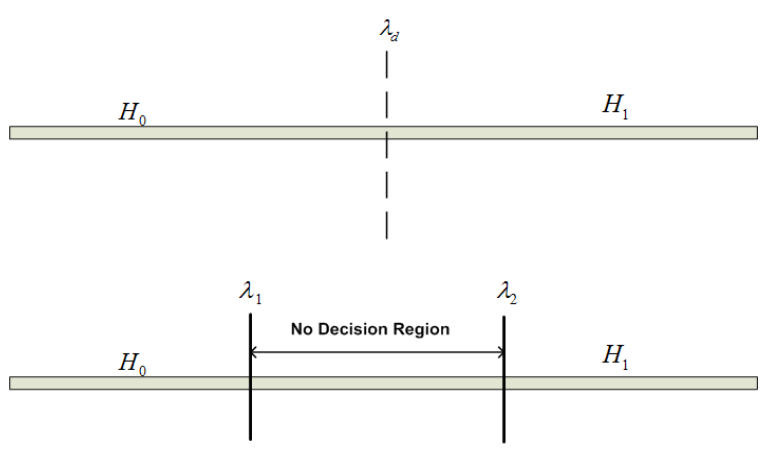

Figure 1. Single threshold vs. double threshold.

\subsection{Conventional Energy Detector}

The signal detection by conventional energy detector (CED) is modeled in $[14,26]$ as,

Hypothesis $0,\left(\mathrm{H}_{0}\right)=$ Primary User absent

Hypothesis $1,\left(\mathrm{H}_{1}\right)=$ Primary User present (1)

The received signal is,

$y(n)= \begin{cases}w(n), & H_{0} \\ x(n)+w(n), & H_{1}\end{cases}$

where $y(n)$ is received signal by $\mathrm{SU}, w(n)$ is the AWGN noise, $x(n)$ is the PU signal. The test statistic is given by,

$$
T_{e d=} \frac{1}{\mathrm{~N}} \sum_{\mathrm{n}-1} \mathrm{~N}|\mathrm{y}(\mathrm{n})|^{2}
$$

where, ' $\mathrm{N}$ ' is the number of samples and ' $\mathrm{T}$ ed ' is the test statistics. The performance metrics for energy detection are

$$
\begin{aligned}
& \mathrm{PD}=\operatorname{Pr}(\text { Ted }>\lambda \mathrm{d} \mid \mathrm{H} 0)=\mathrm{Q}\left(\frac{\lambda \mathrm{d}-\sigma_{\mathrm{w}^{2}}}{\sqrt{\frac{2}{\mathrm{~N}}}(1+\gamma)\left(\sigma_{\mathrm{w}^{4}}\right)}\right) \\
& \mathrm{PFA}=\operatorname{Pr}(\operatorname{Ted}>\lambda \mathrm{d} \mid \mathrm{H} 1)=\mathrm{Q}\left(\frac{\lambda \mathrm{d}-\left(\sigma_{\mathrm{w}^{2}}\right)(1+\gamma)}{\sqrt{\frac{2}{\mathrm{~N}}}(1+\gamma)\left(\sigma_{\mathrm{w}^{2}}\right)}\right) \\
& \mathrm{P}_{\mathrm{MD}}=\left(1-\mathrm{P}_{\mathrm{D}}\right)
\end{aligned}
$$

where, $\lambda_{d}$ is the threshold, $Q($.$) is the Q- function, \gamma$ is the received SNR of the signal, $\mathrm{P}_{\mathrm{D}}$ is probability of detection, $\mathrm{P}_{\mathrm{FA}}$ is the probability of false alarm, $\mathrm{P}_{\mathrm{MD}}$ is the probability of miss detection. The threshold computation as per Neymen- Pearson criterion [20] is,

$\lambda d=\sigma_{\mathrm{w}}^{2}\left(\mathrm{Q}^{-1}\left(\mathrm{P}_{\mathrm{FA}}\right) \sqrt{\frac{2}{\mathrm{~N}}}+1\right)$ 
Recently in [7] authors for the first time utilized the function approximation capability of radial basis function neural network (RBFN) to learn the threshold function for a pre-determined range of $\mathrm{P}_{\mathrm{FA}}$ and $\mathrm{N}$ such that there is no need to compute $\lambda_{d}$ every time and thereby reducing sensing latency.

\subsection{Double threshold based Energy Detection}

In $[4,28]$ it is reported that use of single precise threshold is not accurate under uncertain noise condition, thereby leading to uncertainty in threshold computation. Consequently there is a significant impact on the detection performance under low SNR [3] according to (4) and (5). This motivates the implementation of the double threshold energy detector such that the selected threshold $\lambda_{d}$ of the conventional energy detector is modified as

$\lambda 1,2=\lambda_{d} \pm \Delta \lambda, \lambda 2=\lambda_{d}+\Delta \lambda, \lambda 1=\lambda_{d}-\Delta \lambda$,

where $\lambda$ is the threshold uncertainty and $\lambda_{1}$, $\lambda_{2}$ are the lower and upper bound of threshold $\lambda_{\mathrm{d}}$ such that $\lambda_{1}<\lambda_{2}$. A variation in the range of the uncertainty may give more reliable results compared to a fixed value of the selected threshold which is the motivation to implement the double threshold energy detector with two threshold, $\lambda_{1}$ and $\lambda_{2}$ where $\lambda_{1}<\lambda_{2}$. The $\mathrm{P}_{\mathrm{D}}$ and $\mathrm{P}_{\mathrm{FA}}$ in (4) and (5) are modified as

$\mathrm{PD}=\mathrm{Q}\left(\frac{\lambda_{2}-\left(\sigma_{\mathrm{w}^{2}}\right)(1+\gamma)}{\sqrt{\frac{2}{\mathrm{~N}}}(1+\gamma)\left(\sigma_{\mathrm{w}^{2}}\right)}\right)$

$\mathrm{PFA}=\mathrm{Q}\left(\frac{\lambda_{1}-\sigma_{\mathrm{w}^{2}}}{\sqrt{\frac{2}{\mathrm{~N}}}(1+\gamma)\left(\sigma_{\mathrm{w}^{4}}\right)}\right)$

where $\lambda_{1}$ and $\lambda_{2}$ are the two thresholds. Under this condition of threshold the decision hypothesis for double thresholds is modified as given as

$\mathrm{CD}=\left\{\begin{array}{l}\mathrm{H}_{0}, \mathrm{E} \leq » 1 \\ \mathrm{H}_{1}, \mathrm{E} \geq » 2\end{array}\right.$

According to (11) reliable decision occurs for $\mathrm{H}_{0}$ and $\mathrm{H}_{1}$ while no-decision is taken if the energy values lie between the range $\lambda_{1}$ and $\lambda_{2}$.
$\mathrm{FD}=\{$ NoDecision,$» 1 \leq \mathrm{E} \leq » 2$

This 'no decision' results in sensing failure [29]. To mitigate the above problem, most of the literature deals with Co-operative spectrum sensing [5, 30]. The disadvantages of Cooperative sensing are (a) more sensing time (b) requirement of more physical devices for sensing. Thus, to mitigate existing computational at the local CR level, this paper proposes Neuro-Fuzzy based energy detection scheme to handle system latency and vagueness in computing threshold for spectrum sensing.

\subsection{Proposed NFDT technique}

The proposed scheme of energy detection is shown in Fig. 2 for clarity of understanding to the readers. The proposed detection method first uses the radial basis neural network based function approximation technique [7] to learn the threshold function $\lambda_{\mathrm{d}}$ for a given $\mathrm{P}_{\mathrm{FA}}$ and thereby detect PU signal as per (11). Next an improved fuzzy decision rule base is proposed to account for the decision in region between $\lambda_{1}$ and $\lambda_{2}$ (no decision region shown in Figure $1)$. In doing so, the proposed technique avoid cooperative sensing technique unlike reported in [32] and attain sensing improvement at the local $\mathrm{CR}$ level. If the proposed scheme is adopted in cooperative sensing environment like in [32] then it is expected to give better sensing results due to availability of more information from various CRs as in [32].

\section{Problem Formulation on Fuzzy Decision Rule}

The concept of sensing credibility for spectrum sensing in a cooperative CR environment to improve detection probability for CR networks has been reported in [30]. In [30] credibility information is translated to threshold values $\alpha$ and $\beta$ corresponding to detection probability and probability of false alarm respectively. The present work instead proposes a metric called Fuzzy Credibility to tackle the issue of sensing failure in the 'no decision' region as depicted in Fig. 3. As the energies in the "no decision region' contain certain degree of uncertainty for the occurrence of either hypothesis so one can define a set $\Omega\left\{\mathrm{H}_{0}, \mathrm{H}_{1}\right\}$ and denote this region as $\mathrm{Cr}_{\mathrm{F}}(\Omega)$. Further the uncertain region is divided 
into two equal parts referred as fuzzy credibility and defined as

$\operatorname{Cr}(01)=\frac{E-\lambda_{1}}{{ }_{{ }_{d}}-»_{1}}$

$\operatorname{Cr}(10)=\frac{E-\lambda_{d}}{\lambda_{2}-\lambda_{d}}$

The two metrics in (13) and (14) indicates the measurement of energy possessed by PU signal closer to Hypothesis $H_{0}$ and $H_{1}$ respectively thereby represents in some sense degree of confidence for an undivided confused region [10]. Furthermore, it is pointed out in [22] that sensing credibility is a function of channel condition, which in turn means that the SNR value influences the PU signal. Thus in the present work in addition to two credibility functions $(\mathrm{Cr}(01)$ and $\mathrm{Cr}(10))$, SNR is also included as fuzzy antecedent (input).

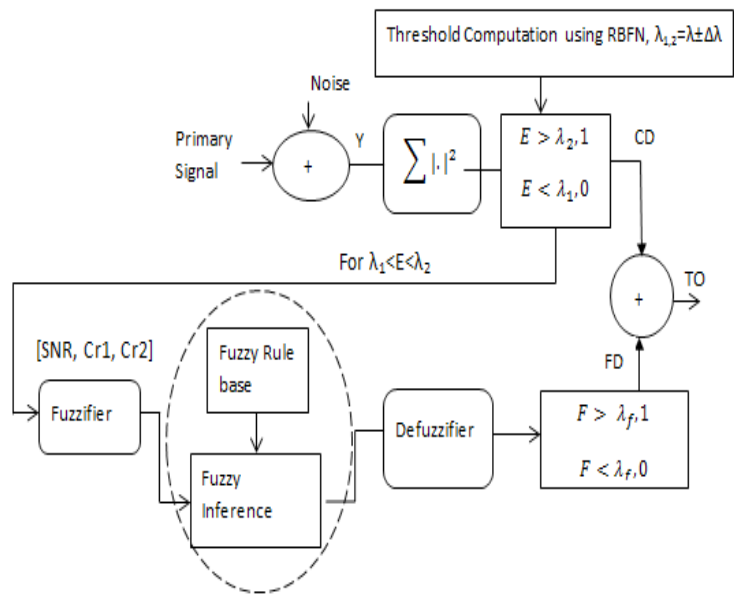

Figure 2. Block Diagram of the proposed model

Based on these three fuzzy inputs, possibility of the presence of PU is selected as Fuzzy output for the confused region (no decision region). The fuzzy linguistic variables along with their membership functions for the fuzzy inputs and outputs are shown in Fig. 4 (a), (b), (c) and (d). The transformation of the fuzzy output (FD), to decision result is done by computing a new threshold called Fuzzy Threshold $\left(\lambda_{\mathrm{f}}\right)$ using newly proposed geometric mean method

$\lambda_{f}=\sqrt[M]{\Pi_{i=1}^{M}\left(F D_{i}\right)}$

The fuzzy output that is obtained after rule aggregation using center of area (COA) method [23] for a particular input, is transformed to the crisp binary decision values $\left\{\mathrm{H}_{0}=0, \mathrm{H}_{1}=1\right\}$ by comparing the outputs with a fuzzy threshold.

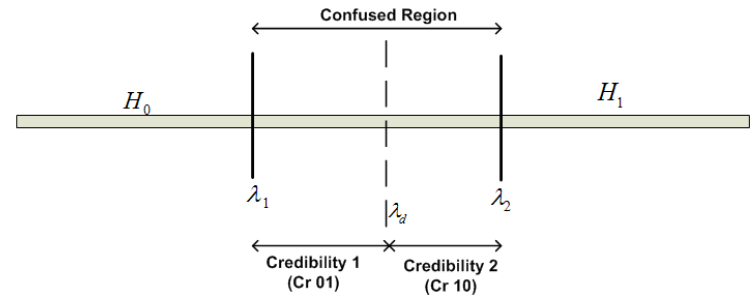

Figure 3. Fuzzy Credibility in confused region

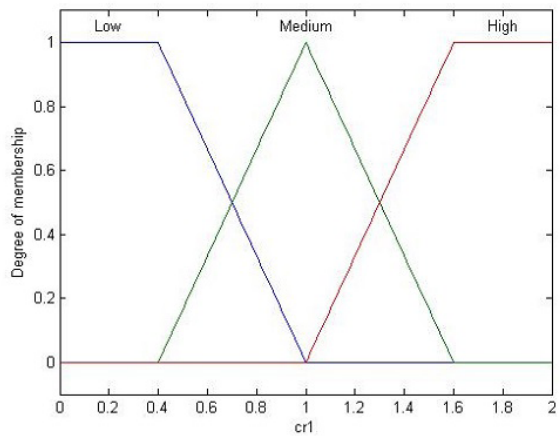

Figure 4(a). Fuzzy MF for Fuzzy credibility $\mathrm{Cr}(01)$

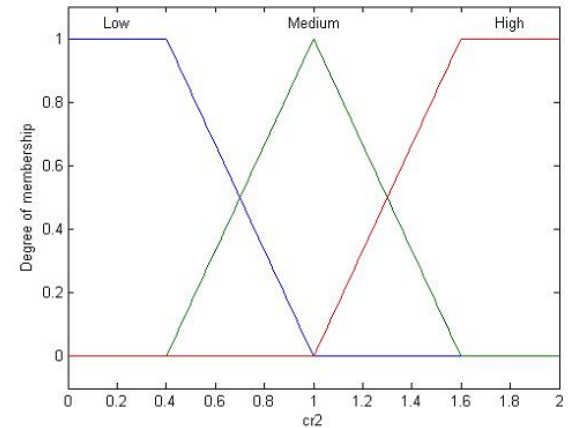

Figure 4(b). Fuzzy MF for Fuzzy Credibility Cr(10)

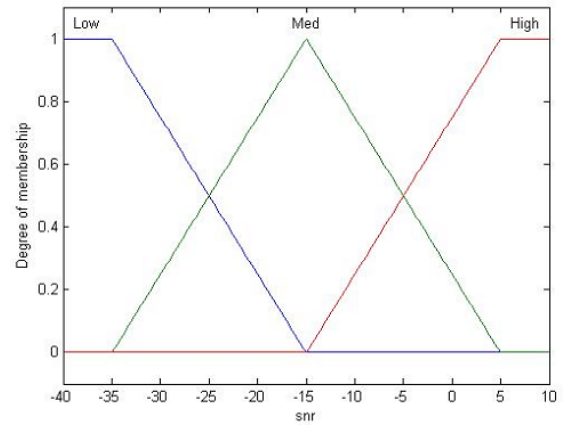

Figure 4(c). Fuzzy MF for SNR (dB)

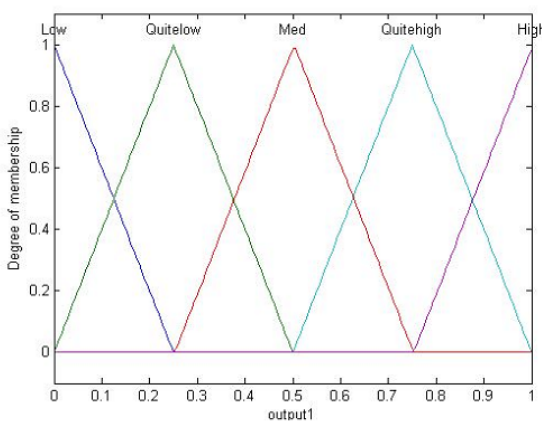

Figure 4(d). Fuzzy MF for output 


\section{Problem Statement on Dynamic Double Threshold}

For practical situations noise variance cannot be estimated accurately and is regarded as an uncertain parameter. Thus here threshold is considered as a function of noise variance and this situation is referred as dynamic thresholding. In this section the second problem related to energy detection using proposed NDFT for the case when $\mathrm{CR}$ system faces uncertain noise variance and hence dynamic double thresholding is presented.

Considering the impact of inaccurate noise variance estimation the threshold function of (7) is modified to dynamic threshold as

$\lambda_{d}^{\prime}=\rho \sigma_{w}^{2}\left(Q^{-1}\left(P_{F A}\right) \sqrt{\frac{2}{N}}+1\right)$

where $\rho$ represent the noise uncertainty factor such that it is close to 1 i.e, $\rho>1$ and $\rho$.

$\lambda_{\mathrm{d}}^{\prime}-\frac{\Delta \lambda}{2}=\lambda_{1}^{\prime}, \lambda_{\mathrm{d}}^{\prime}-\frac{\Delta \lambda}{2}=\lambda_{2}^{\prime}$

On the basis of the above equations the $\mathrm{P}_{\mathrm{D}}$ and $\mathrm{P}_{\mathrm{FA}}$ for fixed double threshold of equation (9) - (10) is modified and is given in (18) and (19).

In most papers it is observed that the calculations of $\mathrm{P}_{\mathrm{D}}$ and $\mathrm{P}_{\mathrm{FA}}$ are done on the basis of equation (18) and (19) without considering the area of no decision region. A decision of the presence or absence of PU signal is given only if the energy values lie outside the no decision region in presence of noise uncertainty. But if the test statistics falls in this region, either this information is ignored for reducing overhead purpose or detection decision is taken in cooperative manner. $\mathrm{P}_{\mathrm{D}}=\lambda_{2}{ }^{\prime} \in\left[\lambda_{2} / \rho, \rho \lambda_{2}\right]^{\min } Q\left(\frac{\lambda_{2}-\left(\sigma_{w^{2}}\right)(1+\gamma)}{\sqrt{\frac{2}{N}}(1+\gamma)\left(\left(\sigma_{w^{2}}\right)\right.}\right)$

$$
=Q\left(\frac{\lambda_{2} / \rho-\left(\sigma_{w^{2}}\right)(1+\gamma)}{\sqrt{\frac{2}{N}}(1+\gamma)\left(\left(\sigma_{w^{2}}\right)\right.}\right)
$$

$P_{F A}=\lambda_{1}{ }^{\prime} \in\left[\lambda_{1} /\right.$

$\left.\rho, \rho \lambda_{1}\right]^{\max } Q\left(\frac{\lambda_{1}-\left(\sigma_{w^{2}}\right)(1+\gamma)}{\sqrt{\frac{2}{N}}(1+\gamma)\left(\left(\sigma_{w^{2}}\right)\right.}\right)=$

$Q\left(\frac{\lambda_{1} \rho-\sigma_{w^{2}}}{\sqrt{\frac{2}{N}}(1+\gamma)\left(\sigma_{w^{4}}\right)}\right)$

This paper proposes PU signal detection when it is within the boundary of the no decision region with the help of Fuzzy logic system exploiting the idea of a double Fuzzy-credibility discussed above.

\section{Proposed Computational Methodology}

In this section computational methodology of the proposed work is presented as outlined in Fig. 2.

(a) First the thresholds $\lambda_{1}$ and $\lambda_{2}$ for the energy detector is computed using pre trained RBFN for a specified probability of false alarm exactly in the similar fashion as discussed in [7]. For easy understanding of the reader the flow chart is presented here in Fig. 5 (Readers are advised to refer [7] for detailed explanation).

(b) Utilizing (11) Monte Carlo simulation is performed for computing probability of detection (hard decision) according to (1). The decision here is referred as 'CD' as shown in Fig. 2.

(c) According to all the energies of PU that lies between threshold $\lambda_{1}$ and $\lambda_{2}$ are left undecided. These energies are collected and processed using proposed Fuzzy rule base as discussed above and depicted in lower portion of Fig. 2. It is worth mentioning at this stage that, the sample space for fuzzy inputs are decided by considering the maximum energy $E_{\max }$ in the no decision region and the gap length of $\mathrm{Cr}(01)$ and $\mathrm{Cr}(10)$ given by

$\frac{\left(E_{\max }, \lambda_{1}<E<\lambda_{2}\right)}{\left(\lambda_{1}-\lambda_{d} \text { and } \lambda_{2}-\lambda_{d}\right)}$

(d) Fuzzy decision 'FD' as shown in Fig. 2 is taken by comparing the fuzzy output $F$ with fuzzy threshold, $\lambda_{\mathrm{f}}$ computed in (15) above.

(e) Finally the total probability of detection (TO) is given by

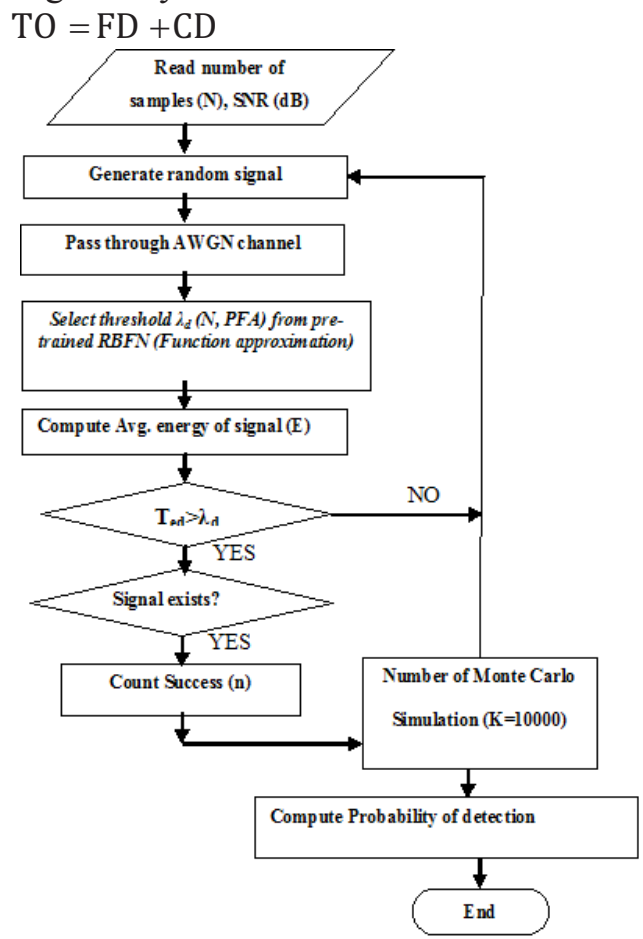

Figure 5. Flow chart of function approximation based energy detection 


\section{Simulation Results}

Scenario 1: The simulation parameters considered here are $\rho=1$ (no noise variance uncertainty) in (16), $\mathrm{N}=1000, \mathrm{SNR}=-10 \mathrm{~dB}$, channel is Additive White Gaussian Noise (AWGN) and the separation between two threshold (No decision region) is $\Delta \lambda=0.1$. The fuzzy decision has been implemented for this scenario using single credibility and double credibility to show the effectiveness of the proposed fuzzy decision rule. Receiver Operating Characteristics (ROC) is shown in Fig. 6.

Scenario 2: This scenario is unlike scenario 1 as in this case $\mathrm{SNR}=-20 \mathrm{~dB}$. With the deterioration in channel condition (or in other words decrease in SNR) both the fuzzy based double threshold technique gives better result than single threshold case of conventional energy detector (CED). It is clear from Fig. 7 that fuzzy rule using double credibility approach outperforms other two methods.

Scenario 3: This scenario is similar to that of scenario 2 except that the separation between $\lambda_{1}$ and $\lambda_{2}$ (of $\left.\Delta \lambda\right)$ has been increased to 0.2 . It is pointed out in $[2,12]$ that increase of $\Delta \lambda$ increases the 'No decision' region thereby resulting in poor sensing performance compared to the above two scenarios.

Scenario 4: This scenario is referred as dynamic double thresholding (D-T) technique due to introduction of uncertainty factor $(\rho)$ along with noise variance term in (16). This scenario makes the fuzzy antecedents (both the credibility) dynamic too thus affecting the fuzzy output in accordance to the uncertainty factor $(\rho)$. The simulation parameters are $\mathrm{N}=1000, \mathrm{SNR}=-20 \mathrm{~dB}$, $\Delta \lambda=0.1$ and $\rho=1.01$.Under dynamic scenario it is found from Fig. 9 that sensing performance at low $\mathrm{P}_{\mathrm{FA}}$ drops down which is quite obvious result.

Scenario 5: This scenario is similar to scenario 4 except that uncertainty factor $\rho$ is reduced here to 1.002 thereby increasing the sensing performance for the proposed method as shown in Fig. 10.

Scenario 6: In this section the efficacy of the proposed method in terms of detection probability with varying SNR condition for uncertain and certain noise conditions are evaluated. It is clear from the Fig. 11 that the proposed method gives better $P_{D}$ values under low SNR condition for both $\rho=1$ and $\rho=1.002$ (certain and uncertain noise conditions) compared to all other methods.

Remark: The $\mathrm{P}_{\mathrm{D}}$ values obtained using proposed method in Fig. 11 for $\mathrm{SNR}=-20 \mathrm{~dB}$ and $\mathrm{N}=1000$ ( $\mathrm{N}$ is the number of samples) are much improved compared to the values reported in [26] for a given $\mathrm{P}_{\mathrm{FA}}$.

\section{Conclusions}

In this paper, a new and improved sensing method is presented at local CR level by providing a solution to the problem of 'no-decision' region under noise uncertainty. Such a CR system give better results compared to conventional double threshold energy detector thereby reducing sensing latency and overall physical system design.

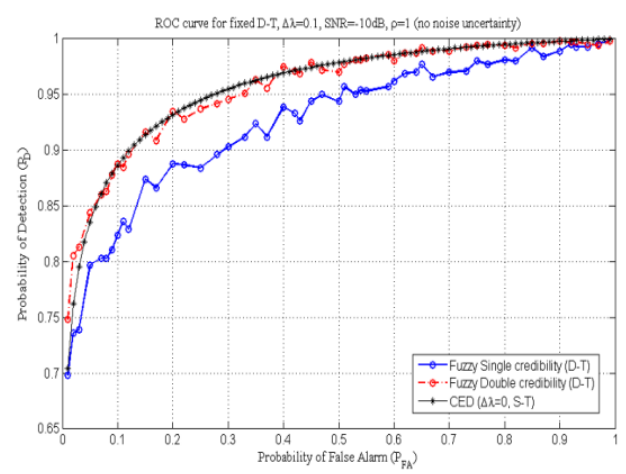

Figure 6. ROC curve for Scenario 1

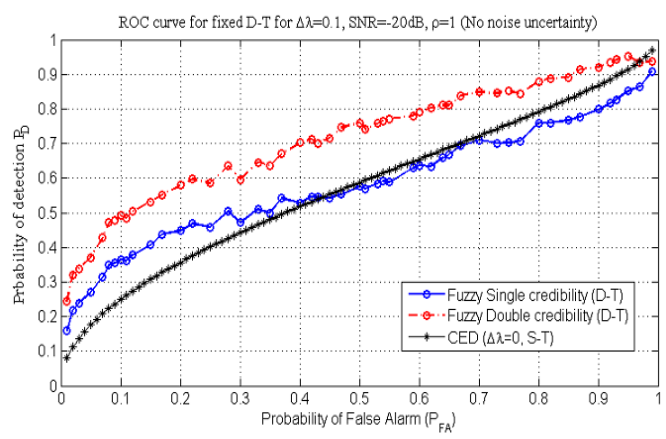

Figure 7. ROC curve for Scenario 2

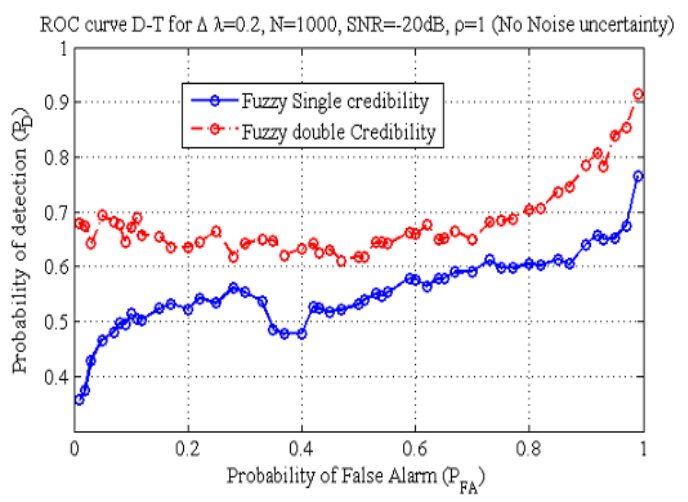

Figure 8. ROC curve for Scenario 3 


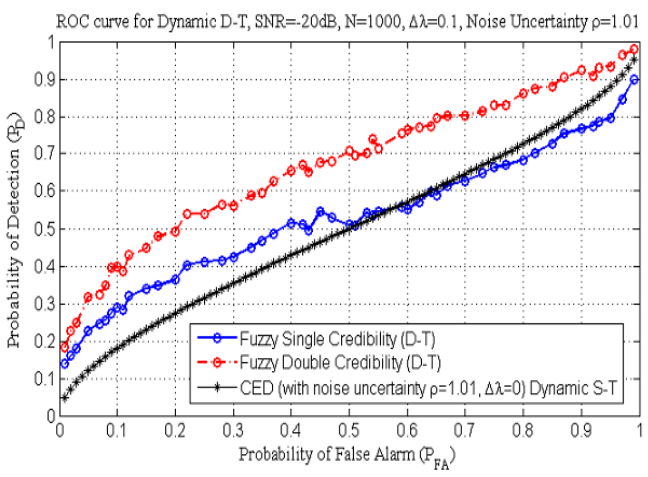

Figure 9. ROC curve for Scenario 4

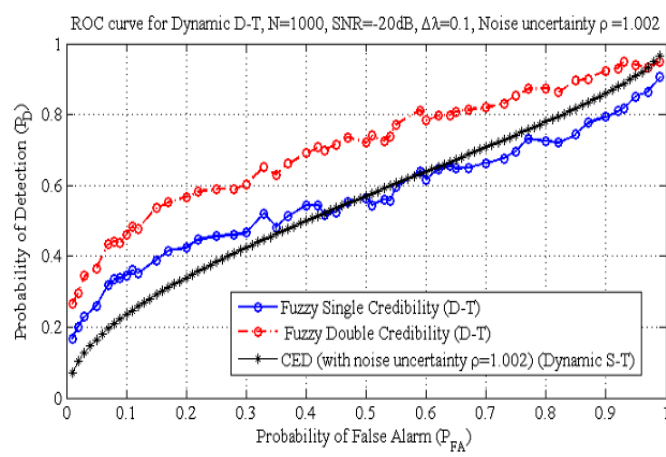

Figure 10. ROC curve for Scenario 5

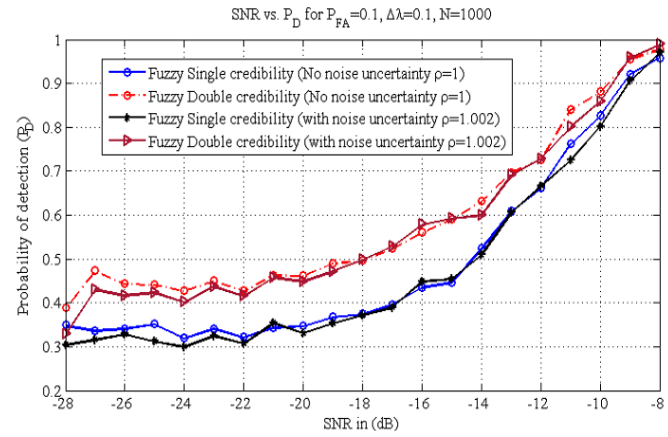

Figure 11. SNR vs. $P_{D}$

\section{REFERECES}

1. Atapattu, S. \& Tellambura, C., Performance of energy detection: A complementary AUC approach. In Proceeding of IEEE Globe Comm, Miami, Florida, USA, December 2010, pp. 1-5.

2. Atapattu, S., Tellambura, C., Jiang, H. (2014). Energy Detection for spectrum sensing in cognitive radio, Springer Briefs in Computer Science, New York.

3. Atapattu, S., Tellambura, C., Jiang, H. (2011). Spectrum sensing via energy detector in Low SNR. Proceedings of IEEE Intl. Conference on Communications, Kyoto, pp.1-5.

4. Alemseged, C, Tran, Y. D., Villardi, H. N., Sun, G., Stanislav, C., F., et al. (2010). Adaptive two thresholds based energy detection for cooperative spectrum sensing." In Proceedings of the IEEE CCNC, pp. 1-6).

5. Bhowmick, A., Chandra, A., Dhar Roy, S., Kundu, S., (2015). Double threshold-based cooperative spectrum sensing for a cognitive radio network with improved energy detectors.IET Communications, Vol. 9 (18), pp. 2216-2226.

6. Bagwari, A.,Tomar, G.S., (2013). Cooperative Spectrum Sensing with Adaptive Double-Threshold Based Energy Detector in Cognitive Radio Networks. Wireless Personal Communication, 73:1005-1019 DOI 10.1007/s11277-013, pp. 1244-3.

7. Dey, B, Hossain, A., Bhattacharjee, A., Dey, R \& Bera, R., (2017). Function approximation based energy detection in cognitive radio using radial basis function network. Intelligent Automation \& Soft Computing, Vol. 23 (3), pp. 393-403.

8. Dandawate, A. \& Giannakis, G., (1994). Statistical tests for presence of cyclostationarity. IEEE Transactions on Signal Processing, Vol. 42, pp. 2355-2369.

9. Ejaz, W., N-ul-Hassan,Aslam S.,\&Kim, H. S. (2011). Fuzzy logic based spectrum sensing for cognitive radio networks. In Proceedings of 5 th IEEE Conference on Next Generation Mobile Applications and Services, pp. 185-189.

10. Ejaz, W., Hattab, G., Attia, T., Ibnkahla, M., (2016). Joint Quantization and confidencebased generalized combining scheme for cooperative spectrum sensing. IEEE Systems Journal,DOI:10.1109/JSYST.2016.2615019, pp. 1-12.

11. Fatty, M.S., Maged, H.I., Ihab, A.A., \& Ibrahim, I.I., (2014). Matched-filter-based spectrum sensing for secure cognitive radio network communications. International Journal of Computer Applications, Vol. 87, pp. 41-46.

12. Giucai,Y. Chengzi, U, L.,Antian, X. M., \& Xi, W. (2012). A novel energy detection scheme based on dynamic threshold in cognitive radio systems. Journal of Computational Information System, Vol. 8, pp. 2245-2252.

13. Haykin, S.(2005). Cognitive radio: Brainempowered wireless communications. IEEE Journal on Selected Areas in Communications, Vol. 23, pp. 201-220.

14. Kalamkar, S. S., \& Banerjee, A. (2013). On the performance of generalized energy 
detector under noise uncertainty in cognitive radio.In Proceeding of National Conference on Communications (NCC), pp. 1-5.

15. Liu, S.Q., Hu, B.J., Wang, X.Y. (2012). Hierarchical Cooperative Spectrum Sensing Based on Double Thresholds Energy Detection. IEEE Communications Letters, Vol. 16 No.7, pp. 1096-1099.

16. Mitola, J, \& MaguireJr. G. Q., (1999). Cognitive radio: Making software radios more personal. IEEE Personal Communications, Vol. 6, pp. 13-18.

17. Mahmood, A.A., \& Hussein, Z.A., (2012). Energy detection technique for spectrum sensing in cognitive Radio: A survey. International Journal of Computer Networks \& Communications (IJCNC), Vol. 4, pp. 223-242.

18. Matinmikko, M., Rauma, T., Mustonen, M., Harjula, I., Sarvanko, H., Mammela, A. (2009). Application of Fuzzy Logic system to Cognitive radio systems. IEICe. Transaction on Commununication, Vol. E93-B No. 12, pp. $3572-3580$.

19. Mohammadi, A., Taban, M.R., Abouei, J.,Torabi, H., (2013). Fuzzy likelihood ratio test for cooperative spectrum sensing in cognitive radio. J. Signal Processing, Vol. 93, pp. 1118-1125.

20. Montazeri, A., Haddadnia, S.H., Safavi, (2016). Fuzzy hypothesis testing for cooperative sequential spectrum sensing under Noise uncertainty. IEEE Communications Letters, Vol. 20, pp. 2542-2545.

21. Mercedes, D., Plataa M., \& Reátiga, Á.G.A. (2012). Evaluation of energy detection for spectrum sensing based on the dynamic selection of detection-threshold. International Meeting of Electrical Engineering ResearchENIINVIE-2012, Procedia Engineering, Vol. 35, pp. 135-143.

22. Qihang, Peng, Kun, Zeng, Wang Jun, Li Shaoqian. (2006). A Distributed Spectrum Sensing scheme based on Credibility and evidence theory in cognitive radio context. IEEE International Symposium on Personal, Indoor and Mobile Radio Communications, Finland, pp. 1-5.

23. Ross, T. (2004). Fuzzy Logic with Engineering Applications. John Wiley and Sons Ltd., USA.
24. Sun, C.H., Zhang, W., \& Letaief, K. Ben. (2007), "Cooperative spectrum sensing for cognitive radios under bandwidth constraints."In Proceedings of the 2007 IEEE Wireless Communications and networking conference, Hong Kong, pp. 1-5.

25. Srivastava, S. K., \& Banerjee, A. (2009). A 'n-ratio' logic based cooperative spectrum sensing using double threshold energy detection. In Proceedings International conference on cognitive radio oriented wireless networks and, communications, Hannover, pp. 312-317.

26. Tanuja S. Dhope, Dina Simunic, Antun Kerner. (2012). Analyzing the performance of Spectrum Sensing Algorithm for IEEE 802.11AF standard in Cognitive Radio Network, Studies in Informatics and Control, Vol. 21(1).

27. Taghavi, E. M., \& Abolhassani, B. (2013) A two step spectrum sensing algorithm using fuzzy logic for cognitive radio networks. International Journal of Communication, Network and Spectrum Sensing, Vol. 4, pp. 507-513.

28. Tandra, R \& Sahai, A., (2008), "SNR walls for signal detection." IEEE Journal of Selected Topic in Signal Processing, Vol. 2(1), pp. 4-16.

29. Verma, P, Singh, B., (2016). Overcoming sensing failure problem in double threshold based cooperative spectrum sensing. Intl. J. Light and Electron Optics, Vol. 121 (9), pp. 4200-4204.

30. Vu-Van, Hiep, Ko, Insoo. (2011). Cooperative Spectrum Sensing with Collaborative Users using Individual Sensing Credibility for Cognitive Radio Network.IEEE Transactions on Consumer Electronics, Vol. 57, No. 2, pp. 320-326.

31. Zeng, Y., Liang, Y-C., Hoang, A. T., \& Zhang, R. (2010). A review on spectrum sensing for cognitive radio: Challenges and solutions. EURASIP Journal on Advances in Signal Processing, Vol. 2010, pp. 1-15.

32. Zhu, J., Xu, Z., Wang, F., Huang, B., Zhang, B. (2008). Double threshold energy detection of cooperative spectrum sensing in cognitive radio. In Proceedings of International Conference on Cognitive radio Oriented Wireless Networks and Communications (CrownCom), Singapore, pp. 1-5. 